\title{
SERUM CYSTATIN C AS A MARKER IN THE ASSESSMENT OF RENAL FUNCTION IN PATIENTS WITH RETINOPATHY AND MILD TO MODERATE DIABETIC NEPHROPATHY
}

\section{ByI}

NADEEJA NIRANJALIE WIJAYATUNGA

Thesis submitted to the University of Sri Jayewardenepura for the award of the degree of Master of Phillosophy in Biochemistry on 8thoff Aprill 2014. 


\section{DECLARATION BY THE CANDIDATE}

The work described in this thesis was carried out by me under the supervision of Professor Hemantha Peiris (Chair professor, Department of Biochemistry, Faculty of Medical Sciences, University of Sri Jayewardenepura), Dr. P.P. Rasika Perera (Senior lecturer, Department of Biochemistry, Department of Biochemistry, Faculty of Medical Sciences, University of Sri Jayewardenepura) and Professor.(Mrs.) Kamani Wanigasuriya (Senior Lecturer, Department of Medicine, Faculty of Medical Sciences, University of Sri Jayewardenepura) and a report on this has not been submitted in whole or in part to any University or any other institution for another Degree/Diploma.

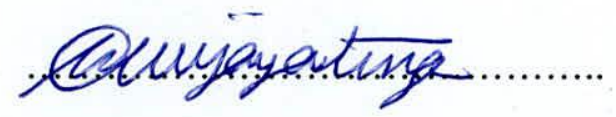

$1.7 / .12 .1 .201 .4 \ldots$ 


\section{Declaration by the supervisors}

We certify that the above statement by the candidate is true and that this thesis is suitable for submission to the University for the purpose of evaluation.

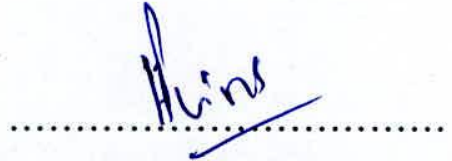

Professor Hemantha Peiris BVSc, MPhil, $\mathrm{PhD}$ (QID.Aus) (Supervisor)

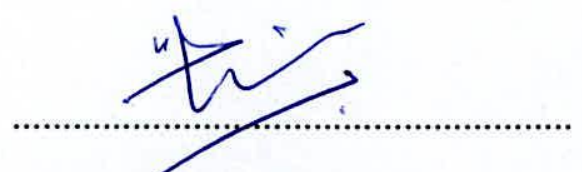

Dr. P.P. Rasika Perera MBBS, $\mathrm{PhD}$

(Supervisor)

\section{Hoanigasua \\ Professor.(Mrs.)Kamani Wanigasuriya \\ MBBS, MD, MPhil, FCCP, FRCP(London), FRACP(Hon.) \\ (Supervisor)}

Date .......7.7./..2../.201.4............. 
We certify that the candidate performed all the corrections as suggested by the examiners.

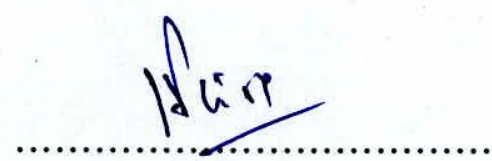

Professor Hemantha Peiris

BVSc, MPhil, $\mathrm{PhD}$ (QID.Aus)

(Supervisor)

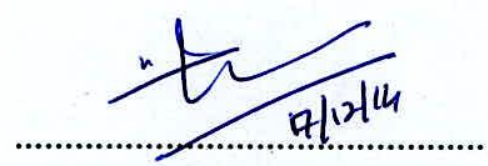

Dr. P.P. Rasika Perera

MBBS, $\mathrm{PhD}$

(Supervisor)

Wtoanigasys

Professor.(Mrs.)Kamani Wanigasuriya

MBBS, MD, MPhil, FCCP, FRCP(London), FRACP(Hon.)

(Supervisor) 


\section{Dedication}

I dedicate this thesis to

my husband, parents, teachers and very close friends 
List of Tables

List of figures iv

Abbreviations vi

Acknowledgement $\quad x$

Abstract xii

1. INTRODUCTION

$\begin{array}{lll}1.1 & \text { General Introduction } & 1\end{array}$

1.2 Chronic Kidney Disease (CKD) 2

1.3 Diabetic nephropathy 2

$\begin{array}{lll}1.4 & \text { Diabetic retinopathy }\end{array}$

1.5 Association between diabetic nephropathy and retinopathy 6

I.6 Investigations currently used in the assessment of diabetic $\begin{array}{ll}\text { nephropathy in clinical practice } & 7\end{array}$

1.6.1 Glomerular Filtration Rate (GFR) 7

1.6.2 Hierarchical arrangement of GFR markers 8

1.6.3 Measured glomerular filtration rate (mGFR) 9

1.6.4 Endogenous markers of Glomerular Filtration Rate 9

$\begin{array}{llr}\text { 1.6.4.i } & \text { Blood urea } & 9\end{array}$

$\begin{array}{ll}\text { 1.6.4.ii } & \text { Serum creatinine } \\ \end{array}$

1.6.4.iii Serum creatinine based estimated Glomerular filtration rates $\quad 12$ 
1.6.4.iii.b.Moification of Diet in Renal Disease study equation 13

1.6.4.iii.c.Chronic Kidney Disease Epidemiology Collaboration Equation 15

$\begin{array}{ll}\text { 1.6.4.iv Twenty four hour urine creatinine clearance } & 16\end{array}$

$\begin{array}{ll}\text { 1.6.5 Albuminuria } & 16\end{array}$

$\begin{array}{lll}\text { 1.6.6 Serum cystatin C } & 19\end{array}$

$\begin{array}{lll}1.7 & \text { Assessment of chronic kidney disease }\end{array}$

$\begin{array}{lll}1.8 & \text { Justification } & 24\end{array}$

$\begin{array}{llr}1.9 & \text { Objectives } & 26\end{array}$

2 LITERATURE REVIEW

$\begin{array}{lll}2.1 & \text { Cystatin C structure and metabolism } & 27\end{array}$

$\begin{array}{ll}2.2 & \text { Factors affecting serum cystatin C levels } 29\end{array}$

2.3 Measurement methods of serum cystatin C 30

$\begin{array}{lll}2.4 & \text { Reference ranges } & 31\end{array}$

2.5 Serum cystatin C as a marker of GFR 33

2.6 Advantages of serum cystatin C over serum creatinine 33

2.7 Serum creatinine and creatinine based eGFR equations $\quad 34$

2.8 Comparison of serum cystatin $\mathrm{C}$ and serum creatinine 36

2.9 Association between serum cystatin C and albuminuria 44

2.10 Prediction of End stage renal failure by renal function markers 46

2.11 Prediction of cardiovascular risk and mortality by renal $\begin{array}{ll}\text { function tests } & 47\end{array}$

2.12 Renal functions and diabetic retinopathy 48 


\section{MATERIALS AND METHODS}

3.1. Chemicals and reagents $\quad \mathbf{5 0}$

$\begin{array}{ll}3.1 .1 & \text { Special chemicals and reagents }\end{array}$

$\begin{array}{lll}3.1 .2 & \text { Water } & 50\end{array}$

3.2 Selection of subjects

3.2.1 Selection of cases (T2DM patients) $\quad 50$

3.2.2 Selection of controls (healthy adults) 51

3.3 Data collection

$\begin{array}{lll}\text { 3.3.1 Questionnaire } & 52\end{array}$

3.3.2 Summary of the data collected and definitions in the type 2

diabetes patients $\quad 52$

3.3.2.i Data collected by interviewing the cases 52

3.3.2.ii Data collected from clinic and past records 53

3.3.3 Summary of the data collected and definitions in the apparently healthy subjects $\quad 54$

3.3.3.i Data collected by interviewing the controls 54

3.3.4 Data collected by measurements in both diabetic patients and healthy adults $\quad 54$

3.3.5 Height, weight and Body Mass Index 55

3.3.6 Waist, Hip circumferences and Waist to Hip ratio 55

3.3.7 Measurement of blood pressure $\quad 56$

3.3.8 Diabetic retinopathy assessment 56

3.4 Collection of blood and urine samples $\quad 56$ 
3.4.1 Collection of blood samples $\quad 56$

$\begin{array}{ll}\text { 3.4.2 Collection of urine samples } & 57\end{array}$

3.5 Laboratory quantitative analysis $\quad 57$

3.5.1. Quantitative analysis of serum cystatin C 57

$\begin{array}{lll}\text { 3.5.1.i } & \text { Cystatin } \mathrm{C} \text { assay procedure } & 57\end{array}$

$\begin{array}{llr}\text { 3.5.1.ii } & \text { Reaction principle } & 58\end{array}$

$\begin{array}{lll}\text { 3.5.1.iii Calibration } & 58\end{array}$

$\begin{array}{llr}\text { 3.5.1.iv Traceability } & 59\end{array}$

3.5.1.v Quality control $\quad 59$

3.5.1.vi Detection limit and measuring range $\quad 60$

$\begin{array}{lll}\text { 3.5.1.vii Imprecision } & 60\end{array}$

3.5.2 Quantitative analysis of serum and urine creatinine 60

$\begin{array}{lll}\text { 3.5.2.i Creatinine assay procedure } & 60\end{array}$

$\begin{array}{lll}\text { 3.5.2.ii } & \text { Reaction principle } & 61\end{array}$

$\begin{array}{lll}\text { 3.5.2.iii Calibration } & 61\end{array}$

$\begin{array}{lll}\text { 3.5.2.iv Traceability } & 62\end{array}$

$\begin{array}{lll}\text { 3.5.2.v Quality control } & 62\end{array}$

3.5.2.v.a. Quality control for serum creatinine $\quad 62$

3.5.2.v.b. Quality control for urine creatinine 63

3.5.2.vi Detection limit and measuring range $\quad 63$

$\begin{array}{lll}\text { 3.5.2.vii Imprecision } & 63\end{array}$

3.5.3 Quantitative analysis of urine albumin 63

$\begin{array}{lll}\text { 3.5.3.i Albumin assay procedure } & 63\end{array}$ 
$\begin{array}{lll}\text { 3.5.3.ii } & \text { Reaction principle } & 64\end{array}$

$\begin{array}{lll}\text { 3.5.3.iii Calibration } & 64\end{array}$

$\begin{array}{lll}\text { 3.5.3.iv Traceability } & 65\end{array}$

$\begin{array}{lll}\text { 3.5.3.v Quality control } & 65\end{array}$

3.5.3.vi Detection limit and measuring range 66

$\begin{array}{ll}\text { 3.5.3.vii Imprecision } & 66\end{array}$

$\begin{array}{lll}3.6 & \text { Calculation of eGFR }\end{array}$

3.6.1 Calculation of eGFR using serum creatinine based Modified Modification of Diet in Renal Disease study equation 66

3.6.2 Calculation of eGFR using serum creatinine based re-expressed Modification of Diet in Renal Disease study $\begin{array}{ll}\text { equation } & 67\end{array}$

3.6.3 Calculation of eGFR using serum creatinine based 2009

CKD-EPI creatinine equation

$\begin{array}{lll}\text { 3.7 Calculation of Albumin to Creatinine Ratio } & 68\end{array}$

$\begin{array}{lll}3.8 & \text { Staging of chronic kidney disease } & 68\end{array}$

3.9 Data processing and analysis $\quad 69$

$\begin{array}{lll}3.10 & \text { Ethical Issues } & \mathbf{7 0}\end{array}$

$\begin{array}{lll}3.10 .1 & \text { Ethical Clearance } & 70\end{array}$

$\begin{array}{lll}3.10 .2 & \text { Consent } & 70\end{array}$

$\begin{array}{lll}3.11 & \text { Sample size calculation } & 71\end{array}$

\section{RESULTS}

4.1 Characteristics of study subjects 
4.2 Associations between serum cystatin $C$ and serum creatinine, albumin to creatinine ratio, and estimated GFR

4.2.1 Association between serum cystatin $\mathrm{C}$ and serum creatinine $\quad 78$

4.2.2 Association of CysC ,SCr, eGFR-MDRD with ACR 80

4.2.3 Association of CysC ,SCr with eGFR-MDRD 83

4.2.4 Comparison between type 2 diabetic patients with mild renal

impairment and moderate renal impairment

4.3 Comparisons of CysC levels in patients with type 2 diabetes and healthy individuals

4.4 Association of diabetic retinopathy with selected renal

function tests

4.5 Establishing normal reference range for serum cystatin $\mathrm{C}$ in a selected sample of healthy Sri Lankan adults

4.6 Analysis of Receiver Operator Characteristics (ROC) curves for determination of diagnostic accuracy of cystatin C in diabetic nephropathy

4.7 Serum cystatin $C$ in levels in identification of diabetic patients with moderate risk of progression of CKD

5 DISCUSSION

6 CONCLUSION

7 REFERENCES 


\section{APPENDICES}

Appendix I - List of publications and communications

Appendix II - Questionnaires- English and Sinhala

153

Appendix III - Consent Forms - English and Sinhala

Appendix IV - Information Sheets - English and Sinhala

Appendix V - Ethics Review Committee letters

Appendix VI - Lever-Jenning charts 


\section{List of Tables}

Page

Table 1.International Clinical Diabetic Retinopathy Disease severity scale

Table 2.Definitions of abnormalities in Albumin excretion according to the 2014

American Diabetes Association Guidelines

Table 3.GFR categories

Table 4.Albuminuria categories

Table 5.Criteria for diagnosis of CKD

Table 6.Different reference ranges for Serum Cystatin C

Table 7.CKD-EPI equations based on Cystatin C or both Serum Creatinine and Serum Cystatin C

Table 8.CKD-EPI equations based on Serum Creatinine levels

Table 9.Characteristics of study subjects (in percentages)

Table 10.Characteristics of study subjects[Mean (SD)]

Table 11.Summary of Hypoglycemic, Antihypertensive and lipid lowering medications used by the type 2 diabetes patients

Table12. Mean (SD) Serum Creatinine, Cystatin C and eGFR values in the study population

Table 13. Summary of correlations between CysC, $\mathrm{SCr}$ and eGFR-MDRD with ACR severity in type 2 diabetes patients and healthy adults

Table 14.Comparison between the Albuminuria categories in type 2 diabetes patients 
Table 15.Comparison between type 2 diabetes patients with mild renal impairment and moderate renal impairment

Table 16.Comparison family history of age and sex matched type 2 diabetes patients and healthy controls

Table 17.Comparison of age \& sex matched type 2 diabetes patients and healthy controls

Table18.Comparison between type 2 diabetes cases with mild to moderate renal impairment with age and sex matched healthy controls

Table 19.Comparison of median (IQ) of Serum Creatinine and Cystatin C of T2DM patients according eGFR and albuminuria categories with matched healthy controls

Table 20.Associations of renal function categories \& Diabetic Retinopathy categories

Table 21.Comparison of diabetes patients with \& without Diabetic Retinopathy

Table 22.Summary of correlation between Retinopathy \& renal function markers

Table 23.Mean (SD) Serum Cystatin C levels according to age categories in healthy adults

Table 24.Comparison of mean Cystatin C levels according age and gender

Table 25.Reference limits and upper and lower limit confidence intervals according to age and gender

Table 26.Diagnostic accuracy at cut-off values of Serum Creatinine and Cystatin C 
Table 27.Diagnostic accuracy at cut-off values of Serum Creatinine and Cystatin C for detection of Albuminuria at ACR $>30 \mathrm{mg} / \mathrm{g}$

Table 28.Comparison of markers of GFR between the low risk and moderate KDIGO risk group 


\section{List of Figures}

Figure 1. Photographs of Retinopathy 5

Figure 2. Prognosis of CKD by GFR and Albuminuria category 23

Figure 3. Models for Cystatin C monomer (a) and dimer (b) 28

Figure 4. Concentrations of Serum Creatinine and Cystatin C according to GFR 34

Figure 5. Cystatin C Calibration curve $\quad 59$

Figure 6. Creatinine Calibration curve $\quad 62$

Figure 7. Urine Albumin MST Calibration curve 65

Figure 8. Categories of Albuminuria according ACR (mg/g) in diabetes patients 77

$\begin{array}{ll}\text { Figure 9. Retinopathy in the diabetes cases } & 77\end{array}$

Figure 10.Scatter plot for Serum Cystatin C \&Serum Creatinine in the diabetes patients

Figure 11. Scatter plot for serum Cystatin C \& Serum Creatinine in healthy controls

Figure 12. Association between Albuminuria categories with Serum Cystatin C and Serum Creatinine in type 2 diabetes patients

Figure 13. Association between Albuminuria categories and estimated GFR calculated using MDRD equation in type 2 diabetes patients

Figure 14.Scatter plot for Serum Cystatin C \& eGFR-MDRD in type 2 diabetes

Figure 15.Scatter plot for Serum Creatinine \& eGFR-MDRD in type 2 diabetes

Figure 16.Scatter plot for Serum Cystatin C \& eGFR-MDRD in healthy controls 
Figure 17.Scatter plot for Serum Creatinine \& eGFR-MDRD in healthy controls

Figure 18.Association of degrees of Albuminuria \& Diabetes Retinopathy status

Figure 19.Normal distribution of Serum Cystatin C in healthy adults

Figure 20.Mean serum Cystatin C levels according to age categories in healthy adults

Figure 21.Serum Cystatin $\mathrm{C}$ values according to the gender in healthy adults

Figure 22.Nonparametric ROC plots to assess the diagnostic efficiency of Serum

Cystatin $\mathrm{C}(\mathrm{CysC})$ and Serum Creatinine ( $\mathrm{SCr}$ ) in diagnosing moderate renal impairment in type 2 diabetes patients

Figure 23. Nonparametric ROC plots to assess the diagnostic efficiency of Serum Cystatin $\mathrm{C}(\mathrm{CysC})$ and Serum Creatinine ( $\mathrm{SCr})$ in diagnosing Albuminuria in type 2 diabetes patients

Figure 24.Serum Cystatin C levels in type 2 diabetes patients according to the

CKD risk categories

Figure 25.Lever-Jenning Chart for Serum Cystatin C Quality Control

Figure 26.Lever-Jenning Chart for Serum Cystatin C Quality Control High

Figure 27.Lever-Jenning chart for Serum Creatinine Quality Control-EXATROL-P 172

Figure 28.Lever-Jenning chart for Serum Creatinine Quality Control-EXATROL-N 172

Figure 29.Lever-Jenning Chart for Urine Creatinine Quality Control Level 1(L1) 173

Figure 30.Lever-Jenning Chart for Urine Creatinine Quality Control Level 2(L2)

Figure 31.Lever-Jenning chart for Urine Albumin Quality Control

Figure 32.Lever-Jenning chart for Urine Albumin Quality Control High 


\section{Abbreviations}

$\mathbf{A}$

ACEi

Angiotensin converting enzyme inhibitor

ACR

Albumin to creatinine ratio

ADA

American Diabetes Association

AER

Albumin excretion rate

ANOVA

Analysis of Variance

ARB

Angiotensin receptor blockers

AUC

Area under the curve

B

BMI

Body mass index

C

CKD Chronic kidney disease

CG

Cockcroft-Gault equation

${ }^{51} \mathrm{Cr}$-EDTA $\quad{ }^{51} \mathrm{Cr}$-ethylenediaminetetra-acetic acid

CVD Cardiovascular disease

CysC Serum cystatin C

D

DR Diabetic retinopathy

DXA Dual energy X-ray absorptiometry

$\mathbf{E}$

eGFR Estimated glomerular filtration rate 
eGFR-MDRD Estimated glomerular filtration rate based on MDRD equation eGFR-CKD EPI Estimated glomerular filtration rate based on CKD EPI equation eGFR-CysC Estimated glomerular filtration rate based on serum cystatin C eGFR-SCr Estimated glomerular filtration rate based on serum creatinine ESRD End stage renal disease

G

GFR Glomerular filtration rate

H

HDL High density lipoprotein

HSD Honestly Significant Difference

I

IFCC International Federation of Clinical Chemistry

K

KDIGO Kidney Disease Improving Global Outcomes

$\mathbf{L}$

LDL Low density lipoprotein

M

macroAU Macroalbuminuria

MAU Microalbuminuria

mGFR Measured glomerular filtration rate

$\mathbf{N}$

NAU Normoalbuminuria 
NHANES III Third National Health and Nutrition examination Survey

NKDEP National Kidney Disease Education Program

NICE National Institutes for Clinical Excellence

NIST National Institute of Standards and Technology

NPDR Non proliferative diabetic retinopathy

$\mathbf{P}$

PENIA Particle enhanced nephelometric immunoassay

PETIA Particle enhanced turbidimetric immunoassay

PDR Proliferative diabetic retinopathy

$\mathbf{R}$

REGARDS Reasons for Geographic and Racial Differences in Stroke

RENAAL Reduction of Endpoints in NIDDM with the Angiotensin II

Antagonist Losartan

ROC Receiver operating curves

$\mathbf{S}$

$\mathrm{SCr} \quad$ Serum creatinine

SD Standard deviation

SPSS Statistical Package for Social Sciences

SRM Standard Reference Material

STDR Site threatening diabetic retinopathy

$\mathbf{T}$

T2DM Type 2 diabetic patients 
${ }^{99 \mathrm{~m}} \mathrm{Tc}-\mathrm{DTPA} \quad{ }^{99 \mathrm{~m}} \mathrm{Tc}$-diethylenethiaminepenta -acetic acid

$\mathbf{U}$

UAC Urine albumin concentration

UAE Urine albumin excretion

UKPDS United Kingdom Prospective Diabetes Study

W

WHR Waist to Hip Ratio 


\section{ACKNOWLEDGEMENTS}

My deepest gratitude goes to my supervisor, Prof. Hemantha Peiris who introduced me to Cystatin $\mathrm{C}$ and motivated me to carry out my research work. His advices, encouragement and guidance are highly appreciated. Also my deepest gratitude is extended to Dr. P.P.Rasika Perera, my second supervisor, who's invaluable, unwavering enthusiasm and mentoring kept me engaged with my research. This thesis would not have been possible without their help, support and patience. I would also like to thank my other supervisor, Prof. Kamani Wanigasuriya, who provided expert advice, guidance and supported me to carry out my research work in the clinical setup. I'm also grateful for Dr. Uditha Bulugahapitiya, Consultant Endocrinologist and the staff of the Diabetic clinic at Colombo South Teaching hospital and also to Dr. M. Wijesuriya the director and the staff of the National Diabetes Center, Rajagiriya for the support given in recruiting patients. I would also like to thank Dr. Ranjith Kodikara, Consultant Eye surgeon and the staff of the Eye clinic, Colombo South Teaching hospital for the invaluable support extended by carrying out eye examinations of the diabetic patients recruited for the study.

I would like to thank the Director and the staff of Colombo South Hospital, Dr. A.L.P. Seneviratne, Head of Department of Family Medicine, Faculty of Medical Sciences, University of Sri Jayewardenepura and the staff of the Family Practice center at University of Sri Jayewardenepura for all the support given in carrying out my research. 
My appreciation also extends to Dr. W.A. Ananda Wijayasiri, Senior lecturer, Department of Community Medicine, Faculty of Medical Sciences, University of Sri Jayewardenepura and Mr. Benoit Ahanda (MSc in Statistics), PhD student, Department of Mathematics and Statistics, Texas Tech University, Lubbock ,Texas for helping me with the statistical analysis.

I also thank the academic and non-academic staff of the Department of Biochemistry, Faculty of Medical Sciences, University of Sri Jayewardenepura for the academic and technical support and assistance since the start of my postgraduate work.

My research would not have been possible without the financial assistance from the University of Sri Jayewardenepura (University grant - ASP/06/R/MED/2011/21) and National Science Foundation, Sri Lanka (Competitive NSF Research grant $\mathrm{RG} / 2011 / \mathrm{HS} / 21)$.

I appreciate all the encouragement and support given by my husband, my parents and friends.

Last but not least, my sincere gratitude is extended to all the subjects who participated very willingly in my study. 


\title{
Serum Cystatin $\mathrm{C}$ as a marker in the assessment of renal function in patients with retinopathy and mild to moderate diabetic nephropathy Nadeeja Niranjalie Wijayatunga
}

\begin{abstract}
Introduction: Serum cystatin $\mathrm{C}(\mathrm{Cys} \mathrm{C})$ has been described as a promising marker of GFR. However there are no literature on performance of $\mathrm{CysC}$ in nephropathy and retinopathy (DR), in Sri Lankan type 2 diabetic patients (T2DM).

Objectives: To determine correlation between serum CysC and Serum Creatinine (SCr), Albumin to Creatinine Ratio (ACR), and estimated glomerular filtration rate based on Modification of Diet for Renal Disease study equation (eGFR-MDRD) in both T2DM patients and healthy adults, to compare CysC levels in T2DM subjects with mild to moderate diabetic nephropathy with age and gender matched healthy individuals and also to identify the correlation of DR in those selected T2DM patients with Cys, SCr, eGFR-MDRD and ACR.

Methods: Sixty one T2DM patients with possibility of mild to moderate renal impairment, and 118 apparently healthy adults, between 30-60 years were enrolled. Out of the 118 healthy adults, 61 were age and gender matched with the T2DM patients. Retinopathy status was assessed by slit lamp examination. $\mathrm{SCr}$ and $\mathrm{CysC}$ and urine creatinine and albumin were measured. ACR and eGFR-MDRD, eGFR- Chronic Kidney Disease Epidemiology Collaboration (CKD EPI) were calculated.

Results: CysC significantly correlated with $\mathrm{SCr}$ and eGFR -MDRD in bothT2DM patients and healthy adults but was stronger in the T2DM patients. CysC significantly
\end{abstract}


correlated with ACR severity categories only in T2DM patients with a significant stepwise increase of CysC according to degree of albuminuria. The T2DM patients $(n=61)$ had higher CysC than the matched controls. Both T2DM patient groups with moderate diabetic nephropathy $(n=21)$ and microalbuminuria $(n=22)$ had higher CysC than the respective matched healthy control groups $(\mathrm{p}<0.05)$. Only the ACR categories showed a significant correlation with $\mathrm{DR}$ categories $(\mathrm{P}<0.05)$. CysC cut off value for the diagnosis of moderate renal impairment was $0.98 \mathrm{mg} / \mathrm{L}$ (sensitivity of $85.7 \%$, specificity of $82.5 \%$ ) and for albuminuria (ACR $>30 \mathrm{mg} / \mathrm{g}$ ) was $0.96 \mathrm{mg} / \mathrm{L}$ (sensitivity of $73.3 \%$ and a specificity of $80.6 \%$ ). Only CysC could differentiate the moderately increased chronic kidney disease (CKD) risk prognosis category from the absent/low CKD risk category $(p<0.05)$. The reference intervals for the healthy adults for $<50$ years of age for male and females are $0.62-1.01 \mathrm{mg} / \mathrm{L}$ and $0.54-0.90 \mathrm{mg} / \mathrm{L}$ respectively while for $>50$ years of age for males and females are $0.65-1.12 \mathrm{mg} / \mathrm{L}$ and $0.60-1.01 \mathrm{mg} / \mathrm{L}$ respectively.

Conclusion: CysC may be used as a reliable renal function marker in T2DM patients with mild to moderate diabetic nephropathy and it is also useful in early detection of poor prognosis in CKD. ACR may be able to predict DR status in T2DM patients. Our study suggests that screening for low GFR with CysC in a low-risk population is probably not worthwhile. In healthy adults, gender based reference intervals for less than 50 year and more than 50 years of age are suggested. 